\section{Serous Meningitis or Aseptic Meningitis}

This is the name given to the syndrome in which a meningeal reaction occurs as the result of an infective focus adjacent to the meninges, e.g. mastoiditis, lateral sinus thrombosis, extradural abscess, intra-cerebral abscess, etc. The pressure of the C.S.F. is increased, and there is an increase in cells, generally polymorphs, but sometimes lymphocytes predominate. The protein is slightly increased, but the sugar and chlorides are normal. The cell count is usually in the neighbourhood of $200-300$, but occasionally it is as high as 1,000 per c.cm. Organisms are not present. In some cases an actual meningitis may supervene.

A lymphocytic meningitis is not an infrequent occurrence in the early stages of Weil's disease and an example came under my own observation recently. The lymphocytes were several hundreds per c.cm. and took two-three weeks to return to normal. There was no jaundice in this patient and the diagnosis was made by finding a high agglutination titre in the blood.

\section{BIBLIOGRAPHY}

ALEXANDER, H. E., 'Treatment of Haemophilus Influenzae Infections, and of Meningococci and Pneumococci Meningitis,' Amer. Four. Dis. Child. Aug., r 943, lxvi, 160.

DINGLE, J. N., FINLAND, M. (1942), War Med., 2, I.

\title{
MENINGITIS: RATIONALE OF DIAGNOSIS
}

\author{
By. JohN APLEY, M.D., M.R.C.P. \\ Department of Child Health, Bristol University
}

Increased power entails increased responsibility. With the advent of chemotherapy delay in the diagnosis of purulent meningitis can no longer be excused, for diagnosis at leisure is condonable only when dealing with an incurable or benign disease. Virtually all the common varieties of purulent meningitis are now curable, yet even now many patients die, or survive only with distressing residua. Inadequacy of laboratory facilities and improper selection of therapeutic agents are contributory factors in the production of these failures ; but the most important single factor is delay in diagnosis.

It is axiomatic in medicine that urgent diagnosis depends on leisurely preparation. The diagnostician who has failed to discipline himself by repeated observation, analysis and synthesis will fail also to attain that maturity of judgment which permits diagnosis to be made rapidly yet without the sacrifice of accuracy. The study of meningitis provides an example of the preliminary, catholic preparation which is essential for urgent diagnosis. Though the present discussion is centred on purulent meningitis the logical approach must be through the subject of lepto-meningitis in its wider aspects.

\section{Features of Meningeal Irritation}

The essential feature common to the many forms of meningitis is meningeal irritation. This phenomenon may occur even in the absence of infection of the meninges; but, whatever the cause of the irritation, the clinical features which may be observed result in all cases from stimula- tion of the meningeal nerves and those portions of the cranial and spinal nerves which traverse the theca. The mechanism is identical with that obtaining in peritonitis, and the results are similar.

The three characteristic features of meningeal irritation are :-(I) pain, (2) hyperaesthesia, and (3) muscular rigidity. The first two of these arise from irritation of sensory components, and the third through the mechanism of a meningealmotor reflex.

The various signs, such as those of Kernig and Brudzinski, and the demonstration of neck rigidity, which are employed in the diagnosis of meningitis, depend for their effects upon increasing the irritability of the meninges by mechanical stretching.

Correlation of Symptomatology with Site of Meningeal Irritation

The production of the three characteristic features of meningeal irritation can be clearly illustrated by contrasting anterior poliomyelitis with meningococcal meningitis. At the same time the discussion can be taken a stage further to provide evidence on which to attempt localization of the site of meningeal involvement.

In anterior poliomyelitis, when the site of predilection is in the lumbar region of the cord, the manifestations of meningeal irritation appear first in the back and the legs ; from these regions they may spread or remain localized, according as the disease process spreads or is halted. In meningococcal meningitis, when, as commonly occurs, the site of predilection is at the base of the brain, the 
results of meningeal irritation are manifest first in the head and neck, to become widespread only as the infective process extends. Since the anterior and middle cranial fossae are supplied by the trigeminal nerve, the results of irritation in these areas will be manifest in the frontal, facial and temporal regions ; the posterior fossa is supplied by the upper cervical nerve roots, so that disturbances originating in stimulation of these nerves will be referred to the occipital and post-nuchal regions. In practice the three fossae are, however, generally involved concurrently, so that clinical localization of the infection to one or other of them is usually not possible.

As between the different types of infective meningitis, the variations in symptomatology may be explained, at least in part, by what is known of their applied pathology. In the meningococcal variety, as also in tuberculous meningitis, the inflammatory exudate tends to be concentrated at the base of the brain. It fills the interpeduncular space, spreading thence with the flow of cerebrospinal fluid down into the spinal theca, forward along the optic nerves, backward into the cisterns, or upward along the middle and anterior cerebral arteries to the vertex. In the natural history of these diseases neck rigidity and occipital pain tend, therefore, to occur at an early stage. In pneumococcal meningitis, on the contrary, the yellow-green exudate may predominate at the vertex of the brain. As a result, though headache is severe, neck rigidity is minimal or absent. In influenzal and in streptococcal meningitis the distribution of the exudate is capricious, following no set pattern, and the symptomatology is correspondingly unpredictable. Post-basic meningitis of infants, a chronic variety of meningococcal meningitis, derives its unique features from the special conditions pertaining in infancy. Because of the comparative softness of the infantile brain the sulci become closed at an early stage in the disease process, so that the exudate is confined almost exclusively to the basal cisterns.

\section{Mechanisms of Production of Meningeal Irritation}

Comprehension of the widely different mechanisms by which meningeal irritation may be produced is of more than academic interest ; without it differential diagnosis remains as unsatisfying as it is superficial. The conditions which give rise to meningeal irritation will be described in five groups, a division which appears natural and contributes to clarity of diagnosis (see Table $\mathrm{I}$ ),

FIG. 1.

PATHOGENESIS OF MENINGEAL REACTIONS

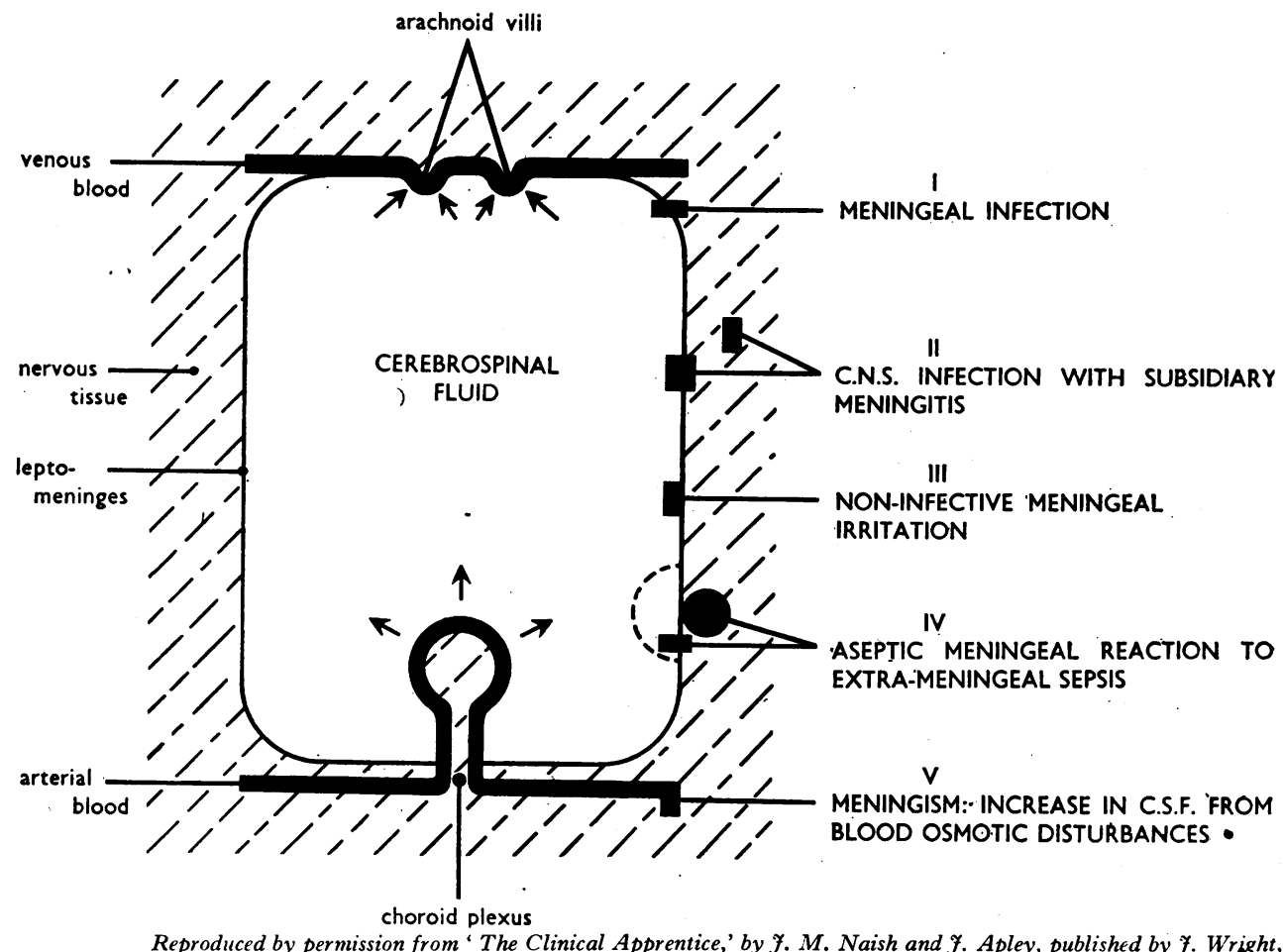

MENINGISM:- INCREASE IN C.S.F. FROM

Reproduced by permission from 'The Clinical Apprentice,' by F. M. Naish and F. Apley, published by $\mathcal{F}$. Wright, Bristol 
TABLE I

\section{Causes of Meningeal Irritation}

Group 1.-Predominantly Meningeal Infections (commonly bacterial).

Cocci : meningo-, strepto-, pneumo-, staphylo-.

Others: B. tubarculosis, $H$. influenzae,

B. coli, B. proteus, Brucellosis, B. typhosus.

Virus : benign lymphocytic meningitis.

Group 2.-C.N.S. Infection with Subsidiary Meningitis (commonly virus).

Anterior poliomyslitis, polioencephalomyelitis, encephalitis (especially in association with specific fevers), infectious mononucleosis.

Syphilis, Weil's disease, actinomycosis and other fungus infections, infections with yeast-like organisms (e.g. torula, coccidioides).

Group 3. Non-infective Mechanical Irritation of Meninges.

Intra-thecal therapeutic agents, e.g. anaesthetics, sera, penicillin, streptomycin.

Blood : Sub-arachnoid or cerebral haemorrhage.

Neoplasm: medulloblastoma, cholesteatoma, oligodendrogliosis, leukaemia.

Chemical : lead encephalopathy.

Cyst fluid.

Group 4. Aseptic Meningeal Reaction to Extra-Meningeal Sepsis.

Skull sepsis, extra-dural abscess, venous sinus thrombosis, brain abscess.

(Otitis media, mastoiditis and sinusitis are the common predisposing causes.)

\section{Group 5. Meningism.}

\section{Infection predominatly meningeal}

In this group are included those conditions in which meningeal irritation is produced by infection confined to, or predominating in the meninges. It has been stated that no organism evokes exclusively a meningitic or an encephalitic reaction, but clinically one or the other almost invariably predominates. In contrast with virus infections, bacterial infections are characterized by a selective affinity for the lepto-meninges, and they produce their effects by direct irritation. It is unnecessary to enumerate all the bacteria which give rise to meningitis; those which have not done so on some occasion must be rare. The bacteria most commonly encountered are shown in Table $\mathbf{I}$, and comprise the common agents of purulent meningitis.

Some virus infections break the general rule, in that the site of maximal infection is in the meninges. Benign lymphocytic meningitis is a common example ; anterior poliomyelitis is also frequently characterized, in its early stages, by a meningeal reaction which is transient.

\section{Infection of C.N.S. with subsidiary meningitis}

In accordance with the general statement previously made, the majority of virus infections of the central nervous system fall into this group. The more important of these are shown in Table $\mathbf{I}$.

Conversely, suppurative encephalitis, though of bacterial origin, is invariably preceded by meningeal inflammation.

Some diseases due to organisms other than viruses also fall into this group. Of these, the only common one is syphilis. Extremely rarely Weil's disease, actinomycosis and some fungus and similar diseases are similarly concerned.

\section{Non-infective mechanical irritation of meninges}

Various non-infective causes may give rise to direct irritation of the meninges. Even normal saline produces a meningeal reaction when introduced into the theca, so that the effects of therapeutic agents similarly introduced can readily be understood. Thus, streptomycin frequently evokes a well-marked pleocytosis (predominantly polymorphonuclear) which may be accompanied by clinical signs of meningeal irritation (M.R.C. I948).

4. Aseptic meningeal reaction to extra-meningeal sepsis

This reaction has been given many names, such as 'aseptic meningitis' and 'sympathetic meningitis'; under the term 'serous meningitis' it is frequently confused with disease-processes which fall more naturally into groups 3 or 5 .

The effects produced arise from irritation by a septic focus which is adjacent, but external to the meninges. In the production of the aseptic meningeal reaction the type of organism does not appear to be of importance. An increase in pressure and of cells in the cerebro-spinal fluid occurs, but the meninges continue to function as a barrier which prevents the passage of organisms. After removal or sterilization of the septic focus the cerebro-spinal fluid reverts to normal. The danger inherent in the situation is that, with failure to recognize and ablate the source of sepsis, the meningeal barrier may be broken down and purulent meningitis result.

\section{Meningism}

The clinical distinction between meningism and meningitis was, according to Walshe, first made by Sir William Jenner. In meningism headache ceases when delirium begins; in meningitis it continues. 
The syndrome of meningism has in the past been attributed to many causes, all equally nebulous, of which the most popular was 'toxaemia.' It now appears clear (Fremont Smith, et al., 1928-29) that meningism is produced, for the major part at least, by disturbances in equilibrium between the blood and the cerebrospinal fluid. The characteristic changes in the cerebro-spinal fluid may occur at the onset of any febrile illness, though only in childhood do signs and symptoms commonly result. When they do occur they are those typical of meningeal irritation, presumably as a direct effect of mechanical stretching of the meninges.

It is important to appreciate the mechanism of production of meningism. It is analogous with the experimental injection of hypotonic solutions into the veins, whereby the osmotic pressure of the serum is reduced, with a resultant increase in the rate of formation of the cerebro-spinal fluid and a rise in its pressure. With the onset of an acute febrile illness the osmotic pressure of the blood falls, and the serum becomes temporarily hypotonic compared with the cerebro-spinal fluid. As a result, there is an increased flow through the choroid plexus, and the cerebro-spinal pressure rises accordingly. The symptomatology is produced by the increase in amount and pressure of the cerebro-spinal fluid; when equilibrium is restored the signs and symptoms disappear.

The common causes of meningism are the common febrile illnesses which have an abrupt onset. It may occur as a transient phenomenon at the commencement of many diseases, but is most marked, and therefore most frequently recognized, in pneumonia, tonsillitis and pyelitis.

\section{Natural History of Purulent Meningitis}

The different mechanisms by which meningeal irritation may be produced, whether in the presence or absence of meningeal infection, have so far been discussed. The complete syndrome of meningeal infection comprises additional components, which can be deduced from general principles.

Purulent meningitis, like tuberculous meningitis, is commonly secondary to infection elsewhere in the body. Before the local effects of infection of the meninges become appreciable the causative agent may produce other disturbances. Usually they are generalized and predominate only at the outset, but this predominance may persist, as not infrequently occurs in infants. They may even prove fatal before meningeal irritation is recognized ; an example is the Waterhouse-Friederichsen syndrome, in which the septicaemic stage of fulminating meningococcal infection may cause death through acute adrenal failure before meningitis becomes clinically manifest.
In its later stages, after the stage of meningeal irritation, with the natural progress of meningeal infection certain mechanical factors may come into operation. As the exudate increases and extends widespread irritative phenomena may occur. Later, organization takes place; obstruction to the flow of the cerebro-spinal fluid may arise, either inside or outside the ventricular system; and the final picture is that of increased intracranial tension, with depression and failure of function.

The stages through which a typical meningeal infection passes are most easily distinguishable in tuberculous meningitis. With purulent meningitis the stages are less clearly demarcated; the sequence may be modified or distorted according to many factors-the characteristics of the causative agent, the rapidity of development of the disease process, the extent and site of maximal infection, the resistance of the subject, and the effects of treatment. The natural history of purulent meningitis, despite these limitations, may however be usefully described according to three stages :-

(I) The stage of invasion.

(2) The stage of meningeal irritation.

(3) The stage of raised intra-cranial tension.

\section{Stage of invasion}

Changes in mentality are often the first to be observed; they may be extremely pronounced or so trifling as to escape notice. The sudden occurrence of delirium accompanied by a rise in temperature is not uncommonly the first manifestation. Irritability alternating with drowsiness is not infrequent. Malaise, discomfort and languor may precede the onset of more definite symptoms by a variable period. Headache, fever and a rise in pulse rate are the rule. Petechial eruptions in the skin are characteristically present in the early stage of meningococcal infection.

The occurrence of vomiting at this early stage is difficult to explain, but it is a common event in pyogenic and, more especially, in tuberculous meningitis.

\section{Stage of meningeal irritation}

The three cardinal features already described are almost always evident. The site at which they appear depends on factors which have been discussed ; but it may be repeated that these manifestations tend to be localized in the early stages, and to become widespread as the infection extends.

Pain is usually described as headache, though there may also be pain down the spine. The headache previously attributable to toxaemia is replaced by one which is more intense. It is described as continous, though fluctuations in 
severity occur ; it may be deep-seated or referred to the surface, usually to the back of the neck and occipital area ; it is aggravated by any movement or manoeuvre which stretches the meninges or increases the-intra-cranial pressure. The shrill ' meningitic cry' may be explained teleologically by a combination of some of these factors ; severe headache compels the patient to cry out, but the cry is restrained in an attempt to limit muscular movement and the consequent rise in intracranial tension which would aggravate the pain.

Local and general hyperaesthesia may be evident early. Muscle rigidity is nearly always demonstrable. In the early stages it may be localized and, since meningitis is commonly maximal at the base of the brain, neck rigidity is an early manifestation, frequently demonstrable when Kernig's, Brudzinski's and other signs are equivocal. Later, the whole back may become rigid, the head retracted, and the thighs flexed on to the abdomen.

\section{Stage of raised intra-cranial tension}

At this stage the classical signs of raised intracranial tension, whatever its origin, may be manifest. Often they occur in an exaggerated form, because of the rapidity with which the tension is increasing ; some, however, may be lacking because of the time factor or for other natural reasons. Headache, for example, may not be registered because the patient is stuporose. Vomiting commonly occurs. Papilloedema is rare except in the late stages of tuberculous meningitis. Drowsiness develops into stupor. The pulse and respiration may become slow and are commonly irregular.

With the spread of the inflammatory process irritative phenomena, such as fits and myoclonic or choreiform movements, may appear, to be succeeded by paralyses. These motor signs tend to develop with involvement of the cerebral vertex. With basal lesions the cranial nerves are more liable to disturbance ; there may be strabismus, pupillary inequality, ptosis, trismus and teeth-grinding.

The terminal phase is one of depression of function leading inexorably to death.

\section{Meningitis in Infancy}

The manifestations of meningitis, producing together so clear-cut a picture in children and adults, are greatly modified in infancy. They may in early life be so atypical as to be misleading unless the great differences are borne in mind. The symptomatology of adult meningitis occurs in infants only at an advanced stage of the disease. Again, the analogy with peritonitis, as contrasted in adults and infants, may be drawn.

In infants in whom the anterior fontanelle re- mains patent, and in the absence of dehydration, palpation reveals an increase of pressure in the fontanelle. This important sign may precede the onset even of neck rigidity by several days. In older children, in whom the fontanelle is no longer patent, there may be no clinical evidence of meningitis beyond fever, failure to thrive, drowsiness alternating with irritability, and in some cases a ' meningitic cry.'

\section{The Cerebro-Spinal Fluid}

Examination of the cerebro-spinal fluid plays an essential part in the diagnosis of meningitis ; it completes, and is complementary to, clinical examination, but cannot be substituted for it. Clinical examination may occasionally afford a complete diagnosis, as in the case of meningococcal meningitis in the presence of meningeal irritation and a petechial rash. As a rule, however, it is concerned in the main with detecting the evidence of meningeal irritation, perhaps among a host of other and confusing data, and with the elimination or disclosure of antecedent conditions. Examination of the cerebro-spinal fluid provides information regarding the nature of any meningeal reaction, and may in some instances furnish an immediate diagnosis. Since the clinical manifesta tions of purulent meningitis are, as a rule, non specific, a specific diagnosis, on which appropriat treatment should be based, must await examination of the fluid.

In the cerebro-spinal fluid the changes associated with meningitis fall into three groups, depending on alteration of function in the meninges and in the vessels of the choroid plexus.

\section{Changes in pressure}

The primary increase in pressure, resulting from increased formation of fluid, may be accentuated later in the disease process by obstruction at those sites from which absorption normally takes place.

With obstruction at any point within the cerebro-spinal system, on the contrary, an apparent diminution in pressure may sometimes result at points distal to the obstruction. In early childhood and in infancy, in particular, obstruction at the base of the brain may result in fluid being obtained under low pressure by the common method of lumbar puncture. In more extreme cases it may prove difficult or impossible to obtain any fluid by this route, though cisternal or ventricular puncture will reveal that the pressure above the site of obstruction is, in fact, considerably raised.

As an aid to diagnosis, changes in pressure must be considered in the light of these findings, and provided always that an adequate technique is adopted for its estimation. On the whole, changes 
TABLE 2

C.S.F. in Meningeal Irritation

1. Pressure 2. Cells 3. Chemistry

(Pressure may be raised in all groups)

\begin{tabular}{|c|c|c|c|c|c|}
\hline \multirow[b]{2}{*}{ Group } & \multicolumn{2}{|c|}{ Predominant Cell } & \multicolumn{2}{|c|}{ Chemistry } & \multirow{2}{*}{ IDENTIFICATION } \\
\hline & $\begin{array}{c}\text { Poly- } \\
\text { morpho- } \\
\text { nuclear }\end{array}$ & $\begin{array}{l}\text { Mono- } \\
\text { nuclear }\end{array}$ & $\begin{array}{l}\text { Sugar and } \\
\text { Chlorides }\end{array}$ & Protein & \\
\hline $\begin{array}{l}\text { I. Bacterial : Mild } \\
\text { Severe } \\
\text { Tuberculous }\end{array}$ & $\begin{array}{l}\text { Increased } \\
\text { Increased }\end{array}$ & Increased & $\begin{array}{l}\text { Normal } \\
\text { Decreased } \\
\text { Decreased }\end{array}$ & $\begin{array}{l}\text { Increased } \\
\text { Increased } \\
\text { Increased }\end{array}$ & $\begin{array}{l}\text { Culture. } \\
\text { Gram stain. Direct typing. } \\
\text { Acid-fast film, culture, guinea-pig } \\
\text { inoculation. }\end{array}$ \\
\hline 2. Virus & & Increased & Normal & Increased & $\begin{array}{l}\text { Complement fixation. Inoculation } \\
\text { in animals. }\end{array}$ \\
\hline $\begin{array}{l}\text { 3. Non-infective Irritation. } \\
\text { (e.g. haemorrhage) }\end{array}$ & Increased & & Normal & Increased & \\
\hline 4. Aseptic Reaction & Increased & & Normal & Increased & Negative culture. \\
\hline 5. Meningism & Normal & Normal & $\begin{array}{l}\text { Normal to } \\
\text { low }\end{array}$ & $\begin{array}{l}\text { Normal or } \\
\text { decreased }\end{array}$ & Primary cause found. \\
\hline
\end{tabular}

in pressure are comparatively valueless in the diagnosis of meningitis. Their occurrence is nonspecific, and the highest pressures are frequently recorded in such conditions as meningism or, fallaciously, through faulty positioning or technique in obtaining the specimen of fluid.

\section{Changes in cells}

In meningism the cells tend to be diminished in number by the process of dilution; in all other types of meningeal irritation a reactionary increase in cells occurs.

The type and number of cells which constitute a pleocytosis depend not so much on the underlying cause as on the speed of onset. Thus, the acute purulent meningitides are almost invariably characterized by polymorphonuclear leucocytosis, while the chronic forms may show a preponderance of lymphocytes. On the other hand, though the usual chronic form of tuberculous meningitis typically shows a lymphocytosis, in its occasional acute form polymorphonuclear cells may predominate. In anterior poliomyelitis a brisk meningeal reaction in the initial stages coincides with a polymorphonuclear leucocytosis ; when the speed of advance of the disease process slackens a lymphocytic reaction occurs.

\section{Chemical changes}

Alteration of the normal chemical composition of the cerebro-spinal fluid may derive from one of two main groups of causes. It may reflect changes in the blood chemistry, even when the meninges are functioning normally ; or it may result from the altered permeability of meninges which are functionally impaired.

Meningism provides an example of the former group. In this condition the cerebro-spinal fluid chlorides are diminished because of the antecedent fall in blood chlorides. The protein in the fluid obtained by lumbar puncture may, however, also be reduced. This reduction results from the enhanced circulation of the cerebrospinal fluid, because with an increased rate of flow the protein in the fluid obtained by lumbar puncture approaches the lower level which is normally to be found only in the ventricles.

Examples of the second group are provided by any infective meningitis. In inflammatory meningitis, and most markedly in acute infections, because of the increased permeability of the blood-cerebro-spinal fluid barrier, the chemical composition of the cerebro-spinal fluid tends to approximate to that of the blood plasma (Cohen, 1927). Those substances normally present in relatively greater quantity in the cerebro-spinal fluid than in the plasma (e.g. chlorides) become reduced in amount; those substances normally present in relatively smaller quantity in the cerebro-spinal fluid (e.g. protein, glucose) become increased. Glucose provides an apparent exception; in meningitis its level in the cerebrospinal fluid may gradually fall, but only because it is utilized in the inflammatory process.

Diagnosis should not rest exclusively on changes in chemical composition, since these changes may 
be considerably delayed. Thus, a normal chloride level is not incompatible with the diagnosis of tuberculous meningitis, for the level may become appreciably reduced only at an advanced stage in the disease process.

Table 2 summarizes the important changes in the cerebro-spinal fluid. It will be seen that variations between the cellular and chemical responses are of great value in diagnosis, though for ultimate identification additional investigations usually prove necessary. The differences in cellular and chemical responses also serve to emphasize the classification of the causes of meningeal irritation prerinusly advanced on an aetiological basis.

\section{Differentiation of Purulent Meningitides}

The optimal treatment of purulent meningitis depends on precise identification of the causal organism. The species and type can be determined only by accurate bacteriological and, where necessary, immunological methods. In the large majority of cases an immediate result can be $\frac{3}{\infty}$ obtained by staining, either of a direct smear or of $\stackrel{\Phi}{\varrho}$ one made after rapid centrifuging of the cerebro- $c$ spinal fluid. With skilled technique and ex- $\overrightarrow{\vec{D}}$ perience the culture of organisms from the fluid in purulent meningitis is more frequently confirmatory than diagnostic. The severity of the infection can rapidly be gauged by a simple quantitative test for glucose concentration.

The identification of haemophilus influenzae by के the above methods is less frequently successful, $\vec{\circ}$ since its morphological features are unpredictably variable. In difficult cases the capsular swelling phenomenon may be employed.

\section{BIBLIOGRAPHY}

COHEN, H. (1927), Brain, L., 601.

FREMONT-SMITH, F., DAILEY, M. E., and THOMAS, G. W. (1928-29), 7. Clin. Invest., 6, 9.

M. R. C. (1948), Streptomycin in Tuberculosis Trials Committee, Lancet, April 17.

\title{
INFANTILE DIARRHOEA AND VOMITING
}

\author{
By IAN M. Anderson, M.D. (Glasgow), M.R.C.P. (London) \\ Assistant Paediatrician, Westminster Hospital
}

Infantile diarrhoea is a disease of infancy characterized by vomiting, frequent watery motions, rapid dehydration, loss of weight and a high mortality rate.

Diarrhoea and vomiting has always been a major problem to those physicians who have had to deal with diseases of children. Epidemics have been reported since early times. In England as long ago as the mid $\mathrm{I} 7^{\text {th }}$ ceritury epidemics of such magnitude occurred as to lead to the death of 2,000 babies during the hot summer weather of I669-7I (Garrison). ${ }^{1}$ Carter ${ }^{2}$ in 1893 drew attention to the infective nature of the disease and noted its relationship to impure milk; he suggested the use of subcutaneous saline. Wilson ${ }^{3}$ (1927) discussed the bacteriology and pointed out that the disease could be caused by many organisms.

Until the second quarter of the present century infantile diarrhoea and vomiting was synonymous with cholera infantum and the main incidence of the disease was in the summer months. Eventually however, investigations showed that contaminated milk and poor hygienic conditions were the most important factors contributing to the seasonal incidence. With improvement in the milk supply, including the use of pasteurized and dried milks and improvement in sanitation associated with the disappearance of the horse, the extermination of flies and the establishment of Welfare Centres where infant hygiene could be taught to the mothers, there was no longer the same seasonal incidence nor mortality. It is probable that a certain amount of infantile diarrhoea and vomiting has always occurred throughout the year and that when the incidence of summer diarrhoea fell below the level of the all-season diarrhoea between rgro and $\mathrm{I} 930$ this latter attracted attention. Marriott ${ }^{4}$ considered that otitis media and mastoiditis commonly caused infantile diarrhoea and vomiting and following on his papers several others apdeared claiming success in treatment by mastoidectomy. Findlay ${ }^{5}$ (1932) discussed the relationship of otitis media to infantile diarrhoea and vomiting and mentioned the social and seasonal incidence. Craig ${ }^{6}$ (1935) drew attention to the disease as it occurred in the newborn and apart from summer diarrhoea and Dick, ${ }^{7}$ Barenberg ${ }^{8}$ and 